\title{
New models for the management of public key infrastructure and root certification authorities
}

\author{
STEPHEN WILSON \\ Associate Director
}

KPMG, Australia

Key words: PKI, certification authority, information security, electronic commerce, certificate

\begin{abstract}
This paper presents a new model for the management of Public Key Infrastructure (PKI), based on standards accreditation, akin to the operation of ISO 9000. In this model, responsibility for certifying end user CAs would rest with Intermediate CAs (ICAs), which in turn would receive their authority from a peak accreditation body. Advantages of this accreditation-based model include its mature and well-respected structure taken from the standards world, a non-government peak authority, clearer definition of the role of the Root CA, more easily understood language for all the elements of the PKI, and clarification of the probable legal liability of all CAs, in particular the peak authority.
\end{abstract}

\section{BACKGROUND ISSUES}

Around the world, PKI rollout has repeatedly stalled over the question of the peak authority or Root CA. National governments have for the most part been reluctant to take responsibility for the peak authority. Their reasons have included fear of the liability thought to go with the top of the 'chain of trust', and philosophical doubts that it is proper or effective to try to 'legislate' trust. Underlying these difficulties is a general lack of clarity about the objectives of PKI and its ability to deliver 'trust'. 
One key obstacle in the debate over the peak authority is that we haven't clearly articulated the functions and benefits of the higher level CAs in the infrastructure; i.e. those CAs that a Root CA certify sign. To be truly useful to relying parties, higher level CAs must do more than merely authenticate other CAs; they should additionally assert the proper operation of those CAs.

Another problem is that certificates are still predominantly thought of as simple electronic passports, identifying the holder to some arbitrary level of confidence. Yet much business is conducted between parties based on their presumed authority or credentials rather than their personal identity. A preoccupation with personal identification within PKI has generally overlooked the routine needs of business transactions and has obfuscated the proper role of the higher levels of the infrastructure.

\subsection{Communities of interest}

Communities of interest are one of the mainstays of the wired world, with rich virtual communities being forged through news and chat groups, and e-mail. But the concept of community is widespread in traditional business and moreover, often has a formal role in the creation of authority and credentials. Many qualifications are directly derived from membership of chartered professional organisations. If the abstraction of identity may be generalised to membership of communities, then identity certificates issued by those communities can serve as electronic credentials. ${ }^{1}$

To support electronic credentials, it is crucial that virtual Certificate Authorities may issue certificates for specific purposes, representing autonomous communities of interest under their own rules of admission or membership. For example, medical registration boards could issue certificates to doctors and law societies could issue certificates to lawyers. Membership of these communities of interest is obviously closed but the intended purpose of the certificates is much more open. All sorts of relying parties will need to have confidence in the validity of the certificates issued to doctors, lawyers and others.

\subsection{The Root CA question}

One of the key issues in building trust in public key systems has been the custodianship of the Root CA. Only a few jurisdictions have so far decided to build central Root CAs (whereas a somewhat higher number of private sector Roots have been established by commercial CAs). Several

${ }^{1}$ It is important to note that any business properties deriving from membership can thereby be represented in an identity certificate issued by the group, without invoking either certificate extensions nor Attribute Certificates. 
administrations have tried to argue that a public sector Root CA is not a core government responsibility, nor is it critical to the success of electronic commerce. $^{2}$ Further, it has been difficult to determine the extent of the Root CA's liability in the event that it is compromised. Yet the expected roles and responsibilities of Root CAs have not been clearly nor generally described, so the debate to date over responsibility and liability has not been altogether rigorous.

A Root CA obviously carries great responsibility for custody of the root private key, for compromise of the root key can theoretically lead to the creation of false CAs and, in turn, unlimited numbers of false certificates. Compromise of a Root CA is perhaps the worst case failure scenario in any PKA scheme. The Root CA must therefore have the highest standards of physical, procedural and personnel security. It is for this reason that most advocates of central Root CAs have guessed that it should be the responsibility of government or perhaps of central banks. In this paper, a robust, highly trusted private sector alternative will be discussed.

Independent of who has custody of the Root CA, note that the existence of intermediate high level CAs in the PKI ameliorates the practical risks arising from compromise. High level CA certificates are issued and revoked relatively infrequently, and neither issuance nor revocation in these cases is needed at short notice. This means the root private key does not need to be on-line or accessible by very many people, making it easier to protect. It is also likely that high level CAs will carry significant intrinsic trust, because they will tend to be large, stable and well known services firms, industry organisations and government units. This means that in the event of a Root CA compromise, the existing high level CAs can probably continue to be trusted. In turn, this obviates the need to revoke all certificates beneath the Root CA.

\section{AN ACCREDITATION-BASED PKI}

Confidence in the validity of certificates boils down to confidence in processes, which in other fields of business usually comes from external audit and certification. This then should be the fundamental role of higher level CAs: independent, third party assurance.

There is a good model for this type of control: the ISO 9000 standard series for management systems. Under ISO 9000 (and indeed a number of

See for example the Australian Government's treatment of a proposed national authentication authority, at www.noie.gov.au/reports/authenticate.html. Some of this author's detailed responses are in the paper "Current issues in the rollout of a National Authentication Framework, under www.acs.org/president/1998/past/io98. 
other international standards) so-called Certification Bodies ${ }^{3}$ conduct periodic audits of organisations to ensure they are complying with their respective management systems. And ISO 9000 certifiers are accredited by national Accreditation Bodies, such as the Registrar Accreditation Board of the US and the Joint Accreditation Scheme of Australia and New Zealand.

\subsection{Defining the role of higher level CAs}

A PKI could be constructed along the same lines if a special type of higher level CA was granted the same sorts of responsibilities as ISO 9000 certifiers. Let us call such a CA an Intermediate $C A$ (ICA). The job of an ICA would be to ensure that end user CAs have procedures in place, fit for the purpose of the certificates they issue, and to monitor ongoing compliance with those procedures. The X.509 certificate issued by an ICA to a user CA is then interpreted explicitly as a compliance certificate, directly analogous to the (paper) certificate issued by an ISO 9000 certifier to a compliant organisation.

This model makes clear the role of the peak authority: it would be to formally accredit ICAs. The role of the Root CA function is also clear. The peak authority needs to be able to digitally sign the accreditation certificates it grants to the ICAs, and the Root CA simply provides that capacity. Thus the Root CA role is quite straightforward. It is clearly delineated from the management responsibility of the peak authority and could be outsourced. And because of the extensive and highly visible processes that attach to accreditation, it would be impractical for bogus ICAs to be spawned, even if the root private key ever were compromised.

\subsection{Comparing roles and responsibilities in ISO 9000}

The diagram below compares the various types of CA (on the right) with the main actors in ISO 9000 (on the left). By convention in the standards world, the various parties are numbered as follows. ${ }^{4}$

The "first party" is a supplier of goods and services. The "second party" is the buyer of those goods and services. The "third party" - a Certifying Body - gives assurance of the quality of the supplier's output by auditing

${ }^{3}$ Note that the term certification has somewhat different meanings in the worlds of standards and of digital certificates. As we shall we, a regular end user Certification Authority usually wouldn't qualify for the description Certification Body.

4 There is an unfortunate inconsistency between the use of the term "third party" in the standards world and the term "trusted third party" as commonly applied in PKI. We may have to live with this. After all, it is no worse than the confusion caused by the co-opting of the term 'certification'! 
the supplier's Quality Management System (QMS) against agreed standards, namely ISO 9000. And the "fourth party" —an Accreditation Body grants Certifying Bodies their authority under the scheme. The ISO 9000 accreditation process references a higher level standard than ISO 9000, namely ISO/IEC Guide 62.

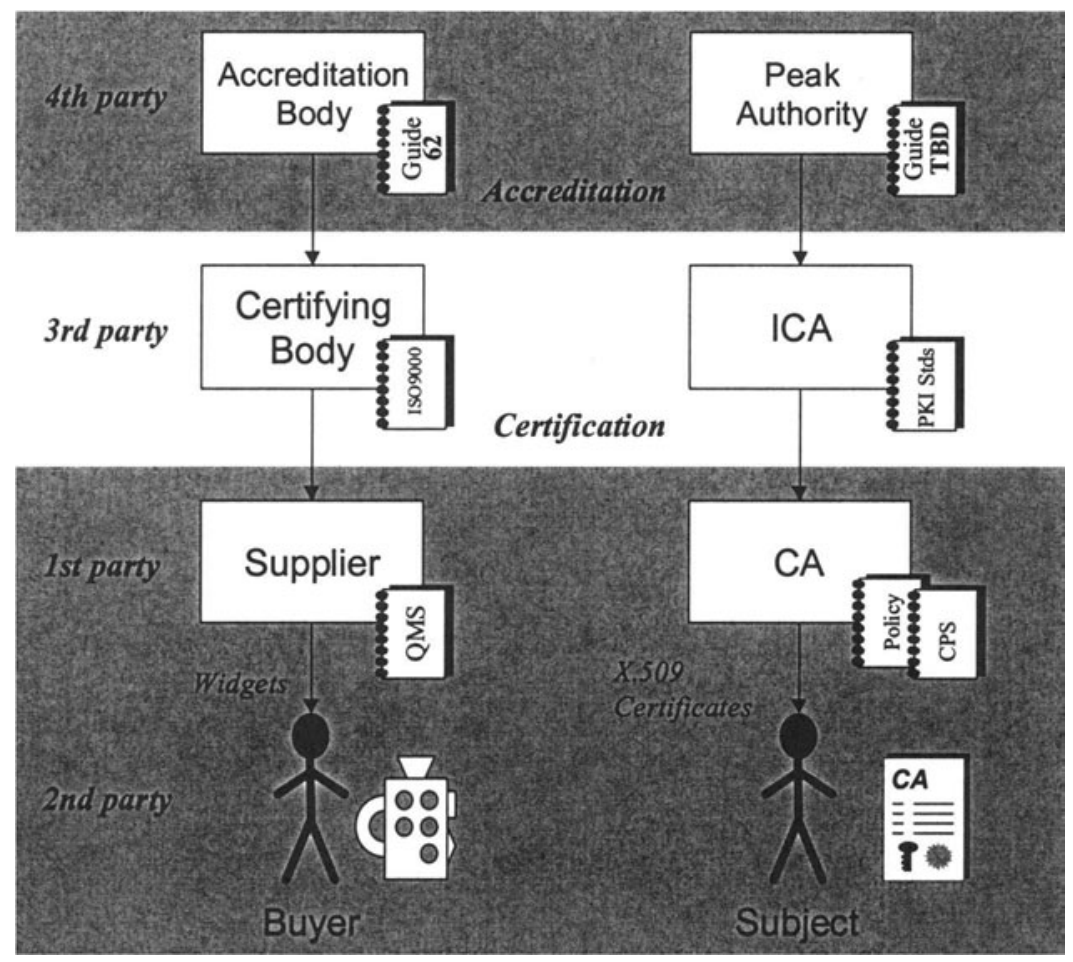

Figure 1. Comparing ISO 9000 and PKI structures

User CAs can be treated as suppliers of a particular type of service product - digital certificates. These are governed by various technical standards, including ANSI X.509, PKCS, and other PKI management standards now in preparation around the world. Corresponding to the supplier's management system under ISO 9000 is a pair of interrelated documents: the CA's Certificate Policy and its Certification Practice Statement (CP/CPS). Together, the CP/CPS specifies what the CA's particular product (digital certificates) is intended for, and defines the process for creating it. We must expect Certificate Policies and Practices to vary from one electronic community of interest to another, just as different management systems are deemed appropriate for different types of conventional products and services. 
ICAs should be given the express role of auditing user CAs against their CP/CPSs. The key underlying question will always be, is the CP/CPS fit for the intended purpose of the certificates? That is, are the processes for vetting applicants well matched to what those people will do with their certificates? And are those processes correctly implemented by the CA? Remember that the meaning of a CA's certificate in this model is an attestation by the ICA not merely that it knows the identity of the CA, but more importantly, that the $C A$ is in compliance.

Likewise, the certificates issued by the peak authority s to ICAs are seen to be digital accreditation certificates, signed by the root key. An unbroken certificate chain from an end user to the Root CA therefore signifies compliance of the user's CA with a Certificate Policy and CPS, fit for the purpose of the user's certificate, whatever that may be.

\subsection{The type of certification and accreditation scheme}

In principle, any industry can decide to make its members subject to management systems standards like ISO 9000 or to industry-specific variants. Alternatively, if there is a more focussed set of technical standards, and if the overhead of ISO 9000 is not warranted, then an industry can institute other types of schemes, including product certification, personnel certification, inspection certification and so on, or hybrids thereof.

There are specific ISO/IEC Guides for the accreditation of certifying bodies in all these types of schemes. The Guides include fundamental generic rules for the conduct of certification, including the need for absolute independence of the certification body (here, ICA) from the business of the certified organisation (i.e. user CA), and the need for established levels of competence within the certifying body.

At this stage, the most appropriate certification for an accreditation-based PKI has not been determined.

\section{HIGHLIGHTS OF THE NEW MODEL}

- It has a "light touch". There need be minimal government involvement in running the scheme. It can leverage an existing international network of accreditation bodies, an established marketplace of certification bodies, and existing ISO/IEC standards.

- The scheme would be optional. There is no general compulsion to have ISO 9000 , although certain customers or even market sectors are free to demand it of their suppliers. Most jurisdictions wish PKI to be similarly optional, in recognition of the UNCITRAL principles of 
technology neutrality, yet allowing for the election of public key technologies where deemed preferable.

- The infrastructure would grow from the bottom up. As with ISO 9000, market forces would drive the uptake of third party assurance. In closed groups or in the early stages of a certificate rollout, operators may choose to run without external assurance. As their communities grow and their interactions become more widespread, market pressure will mount for CAs to obtain certification under ICAs. The economics of external certification will be driven by the value added by third party assurance. CAs would be free to "shop around" for ICAs, which would be expected to compete on the basis of service, expertise in particular sectors, reputation, price and so on.

- Certification would confer no special legal or legislated protection. That is, we need not expect laws to be passed that give preferential treatment to certified CAs, yet demonstrated compliance with best practice, evidenced by a third party audit, is always a good defence against accusations of negligence. Therefore this type of scheme brings generalised legal benefits without requiring specific legislation.

- It "normalises" the liability question for higher CAs and the peak body, bringing more familiar principles to the fore. Liability is well understood in any standards accreditation framework; the risk can even be insured away under errors \& omissions policies (indeed, ISO/IEC Guide 65 requires certifying bodies to carry insurance). In product liability cases affecting ISO 9000 certified companies for instance, unless the auditor was derelict in their duties, they carry limited liability for the actions of the supplier. In practice, liability tends to diminish as you go further up the certification/accreditation chain. It is exceedingly rare for ISO 9000 certifying bodies or accreditation bodies to be sued.

- It allows for fitness for purpose. Just as totally different types of companies can come under the same ISO 9000 management system certification scheme, different CAs could follow their own procedures and membership rules, yet still benefit from PKI compliance. A certificate chain extending from the user CA to the Root CA would signify compliance of the CA with its agreed Policies and Practices no matter what they are - and thus provide relying parties with assurance of the certificates' fitness for purpose.

- It "normalises" the language used in PKI. Relying parties, governments and insurers - to name just a few of the often bewildered parties at present - can now better understand the roles of higher level CAs and the peak authority, because the relationships can be seen as conventional ones of review, audit, accreditation and so on. 
- Finally, it helps to put 'trust' into perspective. Trust is arguably one of the most problematic concepts in information security. Some governments see PKI oversight as equivalent to "legislating trust". But by emphasising fitness for purpose of certificates, PKI can be relieved from the burden of providing open-ended trust. Rather, a certificate should only be seen as evidence that the holder has met the specific rules of some community they represent, and PKI-compliance should only signify that a third party is satisfied that the rules are being properly applied.

\subsection{Reducing the need for cross-certification}

Cross-certification is the practice of mutual recognition of two CA's certificates to an agreed level of confidence. The formal outcome of crosscertification is a "cross certificate" which allows certificate chains beneath the respective CAs to be bi-directionally parsed by relying parties. Legal and technical reviews are required of each others' Policies and Practices, their implementation and operational management, to lead to formal agreement that these are essentially equivalent.

Cross certification remains extremely rare, due to several problems:

- it is a laborious, expensive procedure;

- it does not "scale" for large numbers of CAs (for each pair of CAs which do not share a common higher level CA, the entire cross certification procedure must be repeated);

- cross-certificates are not supported by any current commercial off-theshelf Internet application, so relying parties cannot take practical advantage of the process for processing transactions.

It is commonly believed that cross certification is necessary for effective international certificate-based electronic commerce, and so the problems described above are often seen as serious obstacles. However, this is necessarily not the case. As argued above, if certificates may be associated with membership of separate communities of interest, then their equivalence will usually be entirely moot.

Let us examine independence of certificates in more detail. For Alice and Bob to 'trust' one another in an electronic commerce transaction, it is necessary for them to each have the ability to verify the other's capacity to act. This may necessitate knowing the personal identity of one or both. More commonly in business, the capacity to act will have more to do with credentials and/or position than personal identity. Indeed, in many cases the identity of one or both parties might be suppressed for privacy or personal security reasons (for example, in consumer purchases, voting, legal decisions and police activities). 
Therefore it is reasonable for the parties' certificates to be issued from different CAs or PKIs, and under completely different Certificate Policies and Practices. The real need is not equivalence but rather the ability to verify the capacity to act in a particular transaction.

Note too that in business, it is common for Alice and Bob to act in quite different capacities. For example, they might be a doctor and a patient, or a doctor and an insurance company, or a bank and a customer, or a supplier and a purchasing officer, or a taxpayer and an internal revenue department. In all such cases, if Alice and Bob are to secure their transactions using certificates, their certificates are not supposed to be equivalent. Thus the problem of determining the validity of someone else's certificate is not the same as determining whether or not it is equivalent to your own certificate.

Conventionally, a certificate's validity is verified if it can be traced back to a trusted CA. For a special purpose certificate, representing business credentials such as those indicated above, it is important that the certificate's issuer be independently certified, to show that the certificate is fit for its intended purpose. Under the accreditation-based PKI, such independent certification is evidenced by the digital certificate issued by an ICA.

Therefore, for Alice and Bob to 'trust' one another, they need to trust the source of the credentials that underpin the transaction. If Alice is a doctor, then Bob may need to recognise the medical registration board that issued her certificate. And if Bob is a public health official, then Alice may need to the government department that issued his certificate. Such recognition may simply involve installing the respective issuers' public keys in Bob and Alice's transaction processing systems. There is no need for a cross certificate, and, if Bob and Alice have different roles, then there is actually no possibility of a cross-certificate.

\section{CONCLUSIONS}

This new accreditation-based PKI model leverages existing standards and mechanisms, bringing the realistic prospect of minimalist government involvement yet high levels of integrity and trust. It would appear that national peak authorities can be established relatively easily wherever ISO 9000 or similar schemes are in place.

At the time of writing, discussions had commenced in Australia between the national PKI standards technical committee and the national accreditation body, to determine the best ISO/IEC scheme and to re-scope as required any of the PKI standards already in preparation. 